\title{
Beta-blockers in septic shock to optimize hemodynamics? Yes
}

\author{
Daniel A. Reuter ${ }^{1 *}$, James A. Russell ${ }^{2,3}$ and Armand Mekontso Dessap ${ }^{4,5}$
}

(C) 2016 Springer-Verlag Berlin Heidelberg and ESICM

Septic shock represents one of the maximum physical stresses to the organism. The physiological response to stress includes increased release of catecholamines, leading to a stimulation of cardiac $\beta 1$-adrenergic receptors thereby increasing heart rate and ventricular contractility in order to increase global and microvascular blood flow and oxygen delivery to vital organs. Yet there are adverse effects of adrenergic stimulation including tachyarrhythmias, increased cardiac oxygen consumption with risk of cardiac ischemia, and immune dysregulation. So while it sounds at first contradictory to stabilize the cardiovascular function by giving $\beta$-blocking agents to "brake" the system, there could be benefits. However, is beta-blockade in these clinical circumstances really a brake?

Dr. Morelli and coworkers present in a recent article in Intensive Care Medicine data on a cohort of 45 patients with the primary diagnosis of septic shock, in whom pulling this brake seems to improve cardiovascular function [1]. After initial hemodynamic stabilization over the first $24 \mathrm{~h}$, patients who were tachycardic (heart rate more than $95 \mathrm{bpm}$ ) received a titrated esmolol infusion with the primary goal of reducing heart rate to $80-94 \mathrm{bpm}$ within a time window of $4 \mathrm{~h}$. Indeed, they achieved the intended reduction in heart rate, which could have primarily decreased cardiac output. However, the decreased heart rate was offset by increased ventricular filling time and volume, and decreased left ventricular afterload, ultimately resulting in increased stroke volume, obviously compensating for the decrease in heart rate. Interestingly,

\footnotetext{
*Correspondence: dreuter@uke.de

1 Department of Anesthesiology, Center of Anesthesiology and Intensive Care Medicine, Hamburg Eppendorf University Medical Center, Martinistr. 52, 20246 Hamburg, Germany

Full author information is available at the end of the article
}

For contrasting viewpoints, please go to doi:10.1007/s00134-016-4407-3 and doi:10.1007/s00134-016-4421-5. left ventricular ejection fraction remained unchanged. This, in combination with a decrease in arterial $\mathrm{d} P / \mathrm{d} t_{\max }$ and a concomitant reduction in the need for norepinephrine, strongly points toward a more economical cardiac function under $\beta$-blockade. This mechanism is illustrated in Fig. 1.

Similarly, $\beta$-blockade seemed paradoxical at first but was ultimately shown to be very effective in chronic heart failure [2]. To follow the automobile metaphor, $\beta$-blockade is more a shift to a higher gear, when revolutions per minute are becoming too high. This shift results in the same speed, but with greater fuel efficiency.

So the physiological concept of improving cardiac efficiency seems to work as well in selected patients with septic shock. This is a very important message, since a physiological rationale is one indispensable prerequisite for any new treatment concept. With all enthusiasm, we have to keep in mind that this study performed in a selected group of patients without known cardiac comorbidities was not a randomized controlled trial (RCT), nor was any patient-centered clinical outcome assessed. Second, management of preload has an influence on that treatment concept: the automobile metaphor of changing gears works only with adequate engine cubic capacity. The parallel of engine cubic capacity in patients is cardiac preload that must be in the upper range-otherwise diastolic filling would not increase, when slowing the heart rate-the major prerequisite for ejection fraction to remain stable leading to an increased stroke volume. Morelli and coworkers guaranteed high ventricular preload by keeping central venous pressure $(\mathrm{CVP}) \geq 8 \mathrm{mmHg}$, and pulmonary arterial occlusion pressure (PAOP) $\geq 12 \mathrm{mmHg}$, following the current recommendations for the initial phase of fluid resuscitation in septic shock [3]. Using CVP and PAOP and in particular using those target values for guiding fluid therapy

\section{Springer}



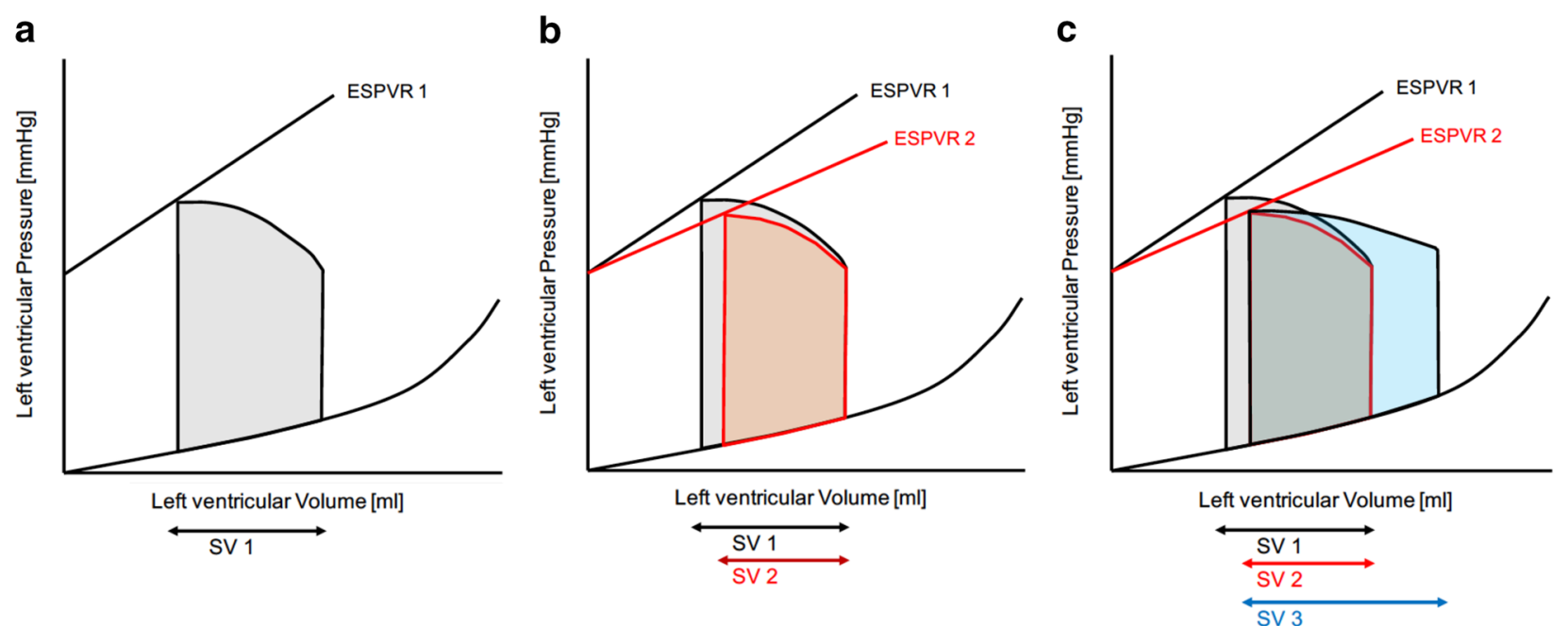

Fig. 1 Pressure-volume loops of the left ventricle are depicted. a Pressure-volume loop prior to $\beta$-blockade; $\mathbf{b} \beta$-blockade leads to a decrease of inotropy, which is reflected by the reduced angle of the ESPVR 2. Accordingly, the resulting SV 2 is diminished; $\mathbf{c}$ if venous return to the left ventricle is high enough, the prolonged duration of diastole caused by heart rate reduction will allow increased left ventricular filling, which, in combination with decreased afterload, will result in an increased SV 3. SV stroke volume, ESPVR end-systolic pressure volume relation

are currently very controversial, primarily because using these targets could lead to fluid overload associated with worse outcome in septic shock $[4,5]$. So the important, conceptual cardiovascular question still needs to be answered: how much preload is necessary for safe and effective $\beta$-blockade in septic shock?

Beta-blockade has been associated with reduced mortality during septic shock in experimental studies and in two preliminary human RCTs [6-8]. A recent small pilot RCT $(n=90)$ in China suggested benefit of the combination of esmolol and milrinone in sepsis for control of heart rate and possibly survival (but the absolute risk reduction was an unlikely $30 \%$ ) [6]. Retrospective cohort studies suggest that chronic use of beta-blockers prior to ICU admission improves short-term survival [9]. A recent systematic review of beta-blockade in sepsis suggests some benefit, but there is still work to do because of the lack of large RCTs-most studies are small and uncontrolled case series/cohorts [7]. In addition to its beneficial effects on cardiac dynamics, beta-blockade may exert beneficial pleiotropic effects including blunting the inflammatory response, metabolic changes, and sepsis-associated coagulopathy [10-14]. Furthermore, beta-blockade may increase microcirculatory/small vessel blood flow in a small cohort $(n=25)$ of patients with sepsis [10]. Interestingly, norepinephrine requirements were significantly decreased by esmolol; however, there was no control group so we cannot be sure these were not just improvements with time.

Animal model studies show marked changes in immune gene expression-primarily anti-inflammatory effects-after $\beta$-blockade with esmolol, so some of the benefit of $\beta$-blockade could be in fact immune-mediated, and not related to the potentially beneficial cardiovascular physiology effects we discussed herein [12, 13]. For example, eight genes with common promoter sequences for NFKB and/or BRCA1 were modulated by esmolol [13]. Analysis of a human database identified the upregulation of CAMP $(p=0.032)$ and TNFSF10 $(p=0.001)$ genes in septic patients compared with healthy controls [13]. In another animal model, esmolol decreased NFKB activation, increased Akt and endothelial nitric oxide synthase phosphorylation, while lowering inducible nitric oxide synthase expression in cardiac and vessel tissues [11]. Esmolol also improves LPS-induced ventricular dysfunction [14]. Thus, esmolol has impressive immune modulation in animal models that may be important in human sepsis, too.

So at this stage, it cannot be anticipated whether this treatment concept will finally lead to an improvement in outcome in real life. However, the therapeutic concept of "setting the brake" by $\beta$-blockade seems deceptively simple and could be effective in carefully chosen septic shock patients (excluding those with hypovolemia, known complex cardiac comorbidities, tachyarrhythmias, hemodynamic instability despite vasopressor treatment, or systolic cardiac dysfunction). These data must stimulate further research, especially well-designed, well-powered RCTs.

\section{Author details}

${ }^{1}$ Department of Anesthesiology, Center of Anesthesiology and Intensive Care Medicine, Hamburg Eppendorf University Medical Center, Martinistr. 
52, 20246 Hamburg, Germany. ${ }^{2}$ Centre for Heart Lung Innovation, St. Paul's Hospital, University of British Columbia, 1081 Burrard Street, Vancouver, BC V6Z 1Y6, Canada. ${ }^{3}$ Division of Critical Care Medicine, St. Paul's Hospital, University of British Columbia, 1081 Burrard Street, Vancouver, BC V6Z 1Y6, Canada.

${ }^{4}$ Assistance Publique-Hôpitaux de Paris, Hopitaux Universitaires Henri Mondor, DHU A-TVB, Service de Réanimation Médicale, 94010 Créteil, France. ${ }^{5}$ Université Paris Est Créteil, Faculté de Médecine de Créteil, Groupe de Recherche CARMAS, 94010 Créteil, France.

Received: 3 May 2016 Accepted: 31 May 2016

Published online: 27 June 2016

\section{References}

1. Morelli A, Singer M, Raieri VM, D'Egidio A, Mascia L, Orecchioni A et al (2016) Heart rate reduction with esmolol is associated with improved arterial elastance in patients with septic shock: a prospective observational study. Intensive Care Med. doi:10.1007/s00134-016-4351-2

2. Packer M, Fowler MB, Roecker EB et al (2002) Effect of carvedilol on the morbidity of patients with severe chronic heart failure: results of the carvedilol prospective randomized cumulative survival (COPERNICUS) study. Circulation 106:2194-2199

3. Dellinger RP, Levy MM, Rhodes A et al (2013) Surviving Sepsis Campaign: international guidelines for management of severe sepsis and septic shock, 2012. Intensive Care Med 39:165-228

4. Cecconi M, De Backer D, Antonelli M et al (2014) Consensus on circulatory shock and hemodynamic monitoring. Task force of the European Society of Intensive Care Medicine. Intensive Care Med 40:1795-1815

5. Boyd JH, Forbes J, Nakada TA, Walley KR, Russell JA (2011) Fluid resuscitation in septic shock: a positive fluid balance and elevated central venous pressure are associated with increased mortality. Crit Care Med 39:259-265
6. Wang Z, Wu Q, Nie X, Guo J, Yang C (2015) Combination therapy with milrinone and esmolol for heart protection in patients with severe sepsis: a prospective, randomized trial. Clin Drug Investig 35:707-716

7. Sanfilippo F, Santonocito C, Morelli A, Foex P (2015) Beta-blocker use in severe sepsis and septic shock: a systematic review. Curr Med Res Opin 31:1817-1825

8. Morelli A, Ertmer C, Westphal M et al (2013) Effect of heart rate control with esmolol on hemodynamic and clinical outcomes in patients with septic shock: a randomized clinical trial. JAMA 310:1683-1691

9. Macchia A, Romero M, Comignani PD et al (2012) Previous prescription of $\beta$-blockers is associated with reduced mortality among patients hospitalized in intensive care units for sepsis. Crit Care Med 40:2768-2772

10. Morelli A, Donati A, Ertmer C, Rehberg S, Kampmeier T, Orecchioni A, D'Egidio A, Cecchini V, Landoni G, Pietropaoli P, Westphal M, Venditti M, Mebazaa A, Singer M (2013) Microvascular effects of heart rate control with esmolol in patients with septic shock: a pilot study. Crit Care Med 41:2162-2168

11. Kimmoun $\mathrm{A}$, Louis $\mathrm{H}$, Al Kattani $\mathrm{N}$ et al (2015) $\beta 1$-adrenergic inhibition improves cardiac and vascular function in experimental septic shock. Crit Care Med 43:e332-e340

12. Dimopoulos G, Theodorakopoulou M, Armaganidis A et al (2015) Esmolol: immunomodulator in pyelonephritis by Pseudomonas aeruginosa. J Surg Res 198:175-184

13. Ibrahim-Zada I, Rhee P, Gomez CT, Weller J, Friese RS (2014) Inhibition of sepsis-induced inflammatory response by $\beta 1$-adrenergic antagonists. J Trauma Acute Care Surg 76:320-327

14. Wang Z, Wu Q, Nie X, Guo J, Yang C (2016) Infusion of esmolol attenuates lipopolysaccharide-induced myocardial dysfunction. I Surg Res 200:283-289 